\title{
Impaired medulloblastoma cell survival following activation of the FOXO1 transcription factor
}

\author{
VINIT KRISHNA SRIVASTAVA, ZIVART YASRUEL and JOSEPHINE NALBANTOGLU \\ Department of Neurology \& Neurosurgery, McGill University; and \\ Montreal Neurological Institute, Montreal, Quebec H3A 2B4, Canada
}

Received April 21, 2009; Accepted June 15, 2009

DOI: 10.3892/ijo_00000419

\begin{abstract}
Medulloblastoma is the most frequent type of childhood brain tumour. The insulin-like growth factor I receptor (IGF-IR) plays a significant neuroprotective role in medulloblastoma survival through regulation of the downstream effectors of the phosphoinositide-3-kinaseprotein kinase-B (PI3K-PKB/c-Akt) pathway. One such target is Forkhead box O1 (FOXO1; FKHR), which is part of the FOXO family of Forkhead transcription factors. Phosphorylation by Akt results in cytoplasmic sequestration of FOXO1 thus inhibiting the expression of genes controlling cell death, cell proliferation, differentiation, cellular metabolism and oxidative stress. Here we show that serum starvation of medulloblastoma cells is accompanied by nuclear translocation of FOXO1. IGF-I stimulation of serum-starved cells resulted in rapid phosphorylation of Akt and FOXO1, and was associated with a significant increase in cell viability. In contrast, expression of a constitutively active form of FOXO1 that cannot be phosphorylated led to a significant reduction in medulloblastoma cell viability, even in the presence of growth factors provided by fetal bovine serum (FBS). These data suggest that the transcription factor FOXO1 may be a critical effector of medulloblastoma growth suppression.
\end{abstract}

\section{Introduction}

Medulloblastoma is a highly malignant tumour of the cerebellum that represents about $20-25 \%$ of all paediatric intracranial neoplasms with the peak incidence occurring between 5 and 9 years of age $(1,2)$. Despite aggressive therapy, which includes surgical debulking, craniospinal irradiation and chemotherapy, the 5-year survival rate hovers around 50-70\% with children under 3 years of age faring much worse $(3,4)$. Furthermore, there are considerable neuropsychological

Correspondence to: Dr Josephine Nalbantoglu, Montreal Neurological Institute, 3801 University Street, Montreal, Quebec H3A 2B4, Canada

E-mail: josephine.nalbantoglu@mcgill.ca

Key words: medulloblastoma, forkhead, FOXO, IGF-I, adenovirus sequelae amongst survivors secondary to both the disease and the treatment (5).

The exact origin of medulloblastoma remains unknown although evidence suggests derivation from the granule cell precursors found in the external germinal layer of the developing cerebellum $(6,7)$. A few potential underlying mechanisms of medulloblastoma initiation and progression have been revealed, which include chromosomal alterations and dysregulation of cell signaling systems $(8,9)$. The PI3K/Akt pathway has been implicated in medulloblastoma cell survival and medulloblastoma tumour formation in humans and in an animal model of the tumour $(10,11)$. The majority of tumour specimens examined to date have shown immunohistochemical staining for activated Akt (phosphorylation of Ser473) $(12,13)$. Furthermore, overexpression of constitutively active Akt in medulloblastoma cell lines reverses the proliferative arrest elicited by the PI3K inhibitor, LY294002 (12). As well, in the $p t c^{+/-}$mice, which spontaneously develop medulloblastoma at a somewhat low frequency (15-20\%), activation of the Akt pathway increases tumour incidence $(10,14)$.

Upon phosphorylation by PI3K, activated Akt can phosphorylate a variety of substrates, including the proapoptotic Bcl-2 family member Bad; caspase-9; nuclear factor-кB; mammalian target of rapamycin; mdm2; and the Forkhead family of transcription factors, FOXO1 (FKHR), FOXO3a (FKHRL1) and FOXO4 (AFX) suggesting a multifactorial mechanism in mediating cell survival through blockade of apoptosis $(15,16)$. In established cell lines, the Forkhead family of transcription factors augments the expression of the cyclin-dependent kinase inhibitor $\mathrm{p} 27^{\mathrm{kip} 1}$ causing cell cycle arrest; further FOXO-induced transcriptional upregulation of FasL and Bim genes can trigger apoptosis (17). Phosphorylation by Akt leads to nuclear export and cytoplasmic retention of FOXO members, preventing activation of gene targets related to growth arrest and apoptosis thereby allowing the target cells to proliferate and/or survive $(15,16)$.

There is currently a dearth of information on the effects of the Forkhead family of transcription factors on the survival of medulloblastoma cells. Consequently, the major aim of this study was to assess the effects of the FOXO transcription factors on medulloblastoma cell survival. We have found that upon serum starvation of the DAOY and UW228-3 human medulloblastoma cell lines, FOXO1 (FKHR) translocates to the nucleus. Furthermore, a constitutively active form of FOXO1 (FOXO1-AAA) that cannot be phos- 
phorylated by Akt was sufficient in significantly reducing medulloblastoma cell viability.

\section{Materials and methods}

Cell lines. The DAOY (American Type Culture Collection; Rockville, MD) and UW228-3 (18) (a gift of Dr Rolando Del Maestro; McGill University, Montreal, Canada) medulloblastoma human cell lines were cultured in $10-\mathrm{cm}$ plates containing Dulbecco's modified Eagle's medium (DMEM) (Gibco-BRL, Grand Island, NY) supplemented with 10\% fetal bovine serum at $37^{\circ} \mathrm{C}$ in a $5 \% \mathrm{CO}_{2}$ atmosphere.

Treatments. Cells were first grown in $10-\mathrm{cm}$ plates until $70-80 \%$ confluency followed by cellular detachment with $5 \mathrm{mM}$ EDTA and plating into 6-well plates at a density of $5 \times 10^{4}$ cells per well in complete serum (DMEM supplemented with $10 \%$ FBS). In order to make cells quiescent, after $24 \mathrm{~h}$, the culture medium was replaced with serum-free medium solution (DMEM supplemented with $0.1 \%$ bovine serum albumin) for $48 \mathrm{~h}$. Cell viability response to IGF-I (Intergen Co., Purchase, NY) by stimulation of quiescent serum starved DAOY cells with 10 and $100 \mathrm{ng} / \mathrm{ml}$ IGF-I, was assessed by a colorimetric assay with the dye (2,3-bis[2-methoxy-4-nitro5-sulfophenyl] $2 \mathrm{H}$-tetrazolium-5-carboxanilide) sodium salt (XTT; Sigma, St. Louis, MO). To investigate the short-term kinetics of phosphorylation of Akt (Cell Signaling Technology, Beverly, MA) and p44/42 MAPK (Cell Signaling Tech.), cells were exposed to IGF-I for 2, 10, 60 and $120 \mathrm{~min}$; cell lysates were subsequently collected for Western blot analysis. To study the effect of IGF-I on the phosphorylation of Akt, p44/42 MAPK and FOXO1 (Cell Signaling Tech.), cells were treated with either LY294002 (25 $\mu \mathrm{M}$; Calbiochem, San Diego, CA) or PD98059 (50 $\mu \mathrm{M}$; Calbiochem) for $2 \mathrm{~min}, 10 \mathrm{~min}, 1 \mathrm{~h}$ and $24 \mathrm{~h}$ following stimulation with IGF-I $(100 \mathrm{ng} / \mathrm{ml})$ for $24 \mathrm{~h}$. Subsequently, lysates were collected for Western blot analysis.

Western blotting. Briefly, cells from different experimental treatment conditions were rinsed in ice-cold PBS and lysed in sample buffer [30 mM Tris ( $\mathrm{pH} 8.0$ ), $150 \mathrm{mM} \mathrm{NaCl}, 1 \mathrm{mM}$ sodium orthovanadate, $1 \mathrm{mM}$ sodium fluoride, $1 \%$ Triton $\mathrm{X}-100,0.5 \%$ sodium deoxycholate, complete EDTA-free protease inhibitor tablet(s)]. Samples with equal amounts of protein were then resolved by denaturing $10 \%$ polyacrylamide gel electrophoresis and electrotransferred to Hybond-ECL nitrocellulose membranes. Membranes were then incubated in $0.1 \%$ Ponceau red to determine protein loadings followed by incubation in 5\% non-fat milk in TBST [20 mM Tris- $\mathrm{HCl}$ (pH 8.0), $150 \mathrm{mM} \mathrm{NaCl}$ and $0.2 \%$ Tween-20] for $1 \mathrm{~h}$ at room temperature and then incubation with the appropriate primary antibody [anti-phospho-Akt (Ser 473), 1:1000; anti-phosphoFOXO1 (Ser 256), 1:1000; anti-Akt, 1:1000; anti-FOXO1, 1:1000; anti-caspase-9, 1:500, Cell Signaling Tech.]; anti-p27, 1:1000 (Santa Cruz Biotechnology, Santa Cruz, CA); anticyclin D1, 1:1000 (NeoMarker, Fremont, CA) and anti-ß-actin, 1:1000 (abcam, Cambridge, UK) at $4^{\circ} \mathrm{C}$ overnight. The membranes were then rinsed thrice in TBST and probed with the corresponding secondary antibodies [anti-rabbit (1:1000; Cell Signaling Tech.)/anti-mouse (1:1000; Dako, Glostrup, Denmark)] conjugated with horseradish peroxidase at room temperature for $1 \mathrm{~h}$. Membranes were finally washed thrice in TBST to remove unbound secondary antibodies prior to revelation using the BLAZE kit (Pierce Biotechnology Inc., Rockford, IL).

Immunofluorescence studies. DAOY cells were plated into wells within the Lab-Tek chamber slide system (Nalge Nunc International, Naperville, IL) at a density of $10^{4}$ cells per well in complete serum. After $24 \mathrm{~h}$, serum starvation was carried out in which the medium was replaced with DMEM (containing $0.1 \% \mathrm{BSA}$ ) for varying times $(1,3,12$ and $24 \mathrm{~h})$. After each time point cells were rinsed twice in ice-cold PBS and fixed with $4 \%$ paraformaldehyde in PBS for $10 \mathrm{~min}$ and permeabilized with ice-cold acetone: methanol (1:1) for $10 \mathrm{~min}$. After blocking with 5\% BSA in PBS containing $0.1 \%$ Triton-X100 for $1 \mathrm{~h}$ at room temperature, primary antibody incubation was performed for $16 \mathrm{~h}$ at $4^{\circ} \mathrm{C}$ using anti-FOXO1 (1:100) diluted in 5\% BSA in PBS. After two washes in PBS, incubation in the appropriate secondary antibody (Alexa Fluor 555; Molecular Probes, OR) diluted in 5\% BSA (1:1000) was done for $1 \mathrm{~h}$ at room temperature. During the last $10 \mathrm{~min}$ of the incubation, Hoechst 33258 (1:1000) was added. Slides were visualized on a Leica DMIRE2 (Richmond Hill, Ontario, Canada) wide-field fluorescence microscope equipped with a CCD camera. The same exposure time was used for all samples. The images were processed using Openlab (Lexington, MA) software. A similar procedure was also utilized for observing FOXO1 translocation during serum-starvation of UW228-3 medulloblastoma cells.

Adenovirus preparation and infection. Ad-FOXO1-AAA (AdFKHR-AAA) was a kind gift of Dr W.R. Sellers (Cambridge, MA). It was generated with the pAD-Easy system described previously (19). Ad-FOXO1-AAA, and Ad-LacZ were amplified in 293A cells and purified by freeze-thaw extraction followed by $\mathrm{CsCl}$ gradient purification. Viruses were titered by monitoring cytopathic effect on 293 A cells. DAOY cells were grown in 10-cm dishes until $80-90 \%$ confluency. These cells were then serum starved for 2 days prior to plating the cells in 6-well plates containing serum-free medium. Selected wells were supplemented with IGF-I $(100 \mathrm{ng} / \mathrm{ml})$ and allowed to grow for $24 \mathrm{~h}$ at $37^{\circ} \mathrm{C} / 5 \% \mathrm{CO}_{2}$. Subsequently, Ad-FOXO1-AAA was added at MOI 100 and the control Ad-LacZ was added at the same MOI to ascertain the possibility of non-specific viral toxicity. DAOY cells were exposed to the viral treatment for 24,48 and $72 \mathrm{~h}$ at $37^{\circ} \mathrm{C} /$ $5 \% \mathrm{CO}_{2}$. For each time-point, cell viability was assessed with the XTT assay (Sigma). For adenoviral transduction into $10 \%$ FBS containing media, DAOY or UW228-3 cells were first counted and allowed to adhere for about $6 \mathrm{~h}$ prior to transduction with Ad-FOXO1-AAA, Ad-LacZ or Ad-BFP at their respective MOIs. Both medulloblastoma cell lines were exposed to viral treatment for 24,48 and $72 \mathrm{~h}$ at $37^{\circ} \mathrm{C} /$ $5 \% \mathrm{CO}_{2}$ prior to XTT viability assessment.

\section{Results}

FOXO1 undergoes nuclear translocation during serum starvation. Medulloblastoma cell lines in culture are sensitive to serum deprivation. In both the DAOY and UW228-3 
A

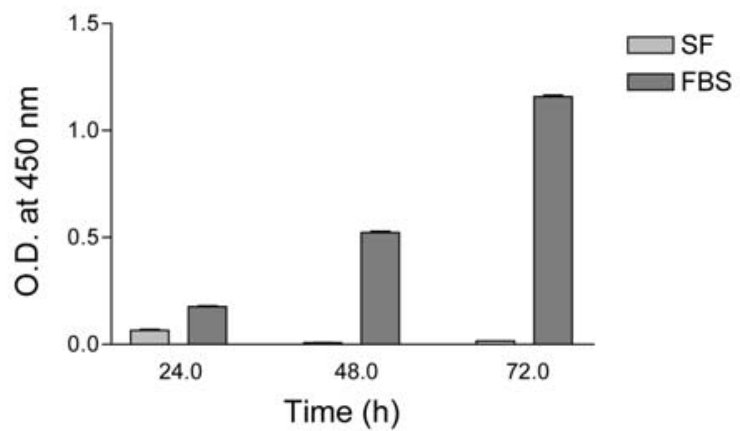

B

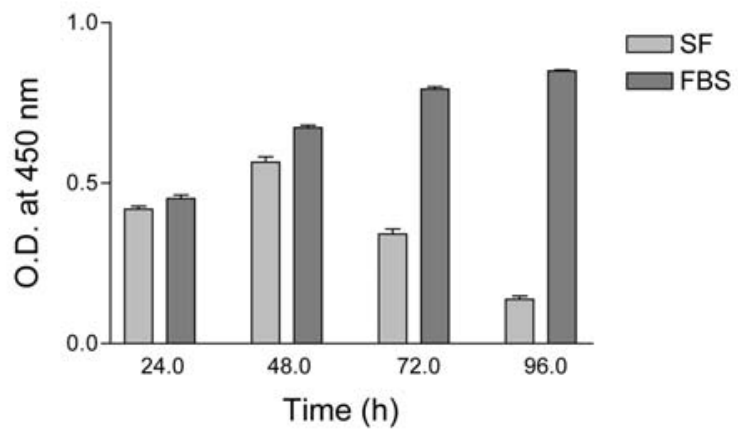

Figure 1. Serum starvation decreases both (A) DAOY and (B) UW228-3 medulloblastoma cell viability. The cells were plated at $5 \times 10^{4}$ cells/well in the presence of $10 \%$ FBS in a 6-well plate. The cells were then buffer exchanged into serum-free (SF) medium and cell viability was assessed at 24, 48, 72 and $96 \mathrm{~h}$ using the XTT assay. The results are representative of three experiments and are shown with standard deviation. cell lines, upon withdrawal of serum, metabolic activity diminishes and cell numbers decrease (Fig. 1).

As the PI3K/Akt pathway has been implicated in medulloblastoma survival, we examined a distal target of the PI3K/Akt pathway, FOXO1 during serum starvation of DAOY and UW228-3 cell lines. FOXO1 was chosen as a representative of the FOXO family of Forkhead transcription factors, which include FOXO1, FOXO3a and FOXO4. To examine the localization of FOXO1 transcription factor upon growth factor withdrawal, DAOY cells were placed into serum-free media for varying periods of time. Endogenous FOXO1 was primarily confined to the cytoplasm of DAOY cells proliferating in $10 \%$ fetal bovine serum (Fig. 2). Transfer of these cells to serum-free medium [containing $0.1 \%$ bovine serum albumin (BSA)] resulted in FOXO1 re-localization to the nucleus after $24 \mathrm{~h}$ with progressively stronger FOXO1positive immunoreactivity being observed within Hoechstpositive nuclei (Fig. 2A). Analysis of 6 high-power fields (hpf) per time point revealed that most of the FOXO1positive nuclei are observed after $24 \mathrm{~h}$ (Fig. 2A). Nuclear FOXO1 immunoreactivity was also observed in serum-starved UW228-3 medulloblastoma cells, which displayed reduced viability (Figs. 1 and 2 C). These results show that serum starvation of the medulloblastoma cells is accompanied by nuclear translocation of FOXO1.

Expression of IGF-IR and IGF-I-dependent survival signaling in human medulloblastoma cells. The insulin-like growth factor I (IGF-I) is a potent survival factor which signals through
A

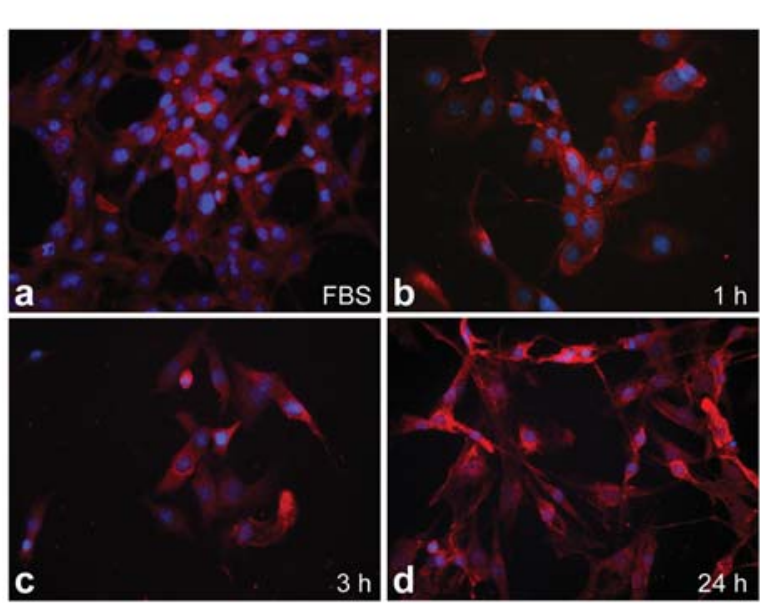

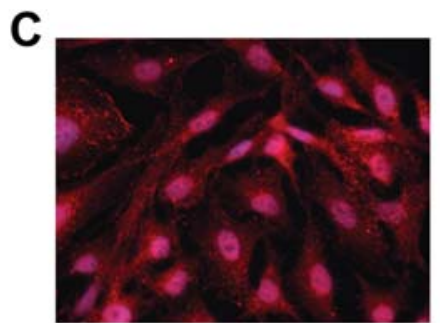

${ }^{*} \mathrm{P}<0.001$

B

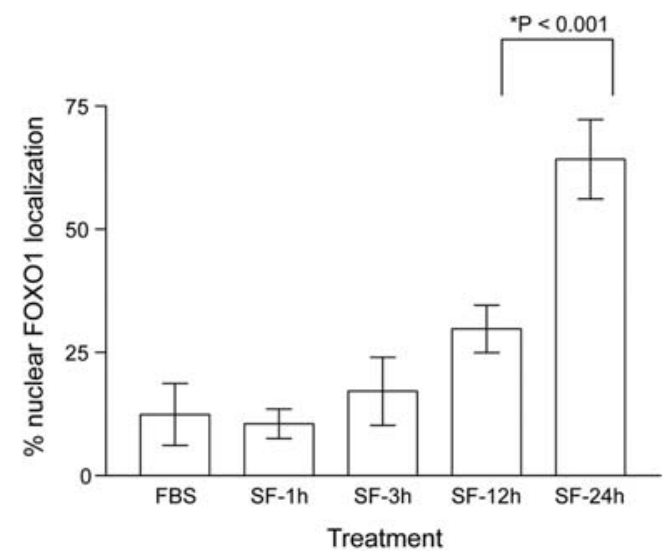

Figure 2. FOXO1 nuclear localization upon serum-starvation of DAOY and UW228-3 medulloblastoma cells. (A) DAOY cells were serum starved for varying times (1,3 and $24 \mathrm{~h}$ ) followed by fixation and permeabilization (a-d). Subsequently, cells were stained with anti-FOXO1 (Alexa Fluor 555; red) and Hoechst 333248 (nuclear stain; blue) and images were captured with a fluorescent microscope as described in Materials and methods. (B) Graphical representation of FOXO1 nuclear localization in DAOY medulloblastoma cells; bars represent mean \pm SEM from at least six independent high-power field. P-value was determined by using One-way ANOVA with Bonferroni's multiple comparison post-test. (C) Endogenous FOXO1 nuclear localization in UW228-3 cells after $24 \mathrm{~h}$ serum starvation as visualized by $63 \mathrm{x}$ oil immersion. 
A

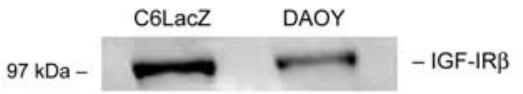

B
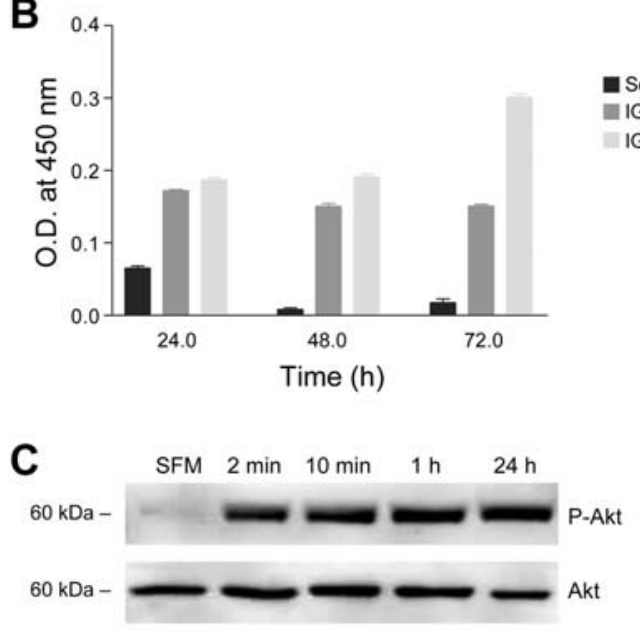

D

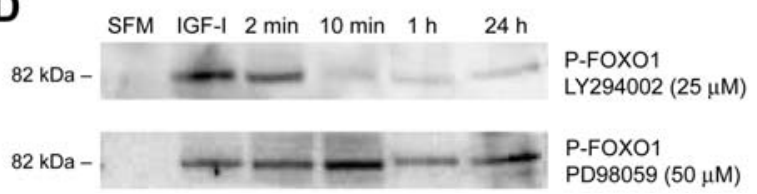

Figure 3. Effect of IGF-I on medulloblastoma cell survival. (A) Expression of IGF-IRß in the DAOY medulloblastoma cell line; C6LacZ was used as a positive control for IGF-IRß expression. (B) IGF-I promotes survival of DAOY medulloblastoma cells under serum-free conditions. Complete serum (10\% FBS) was replaced with serum free medium (SF) and cells were cultured for an additional $48 \mathrm{~h}$ under SF conditions. Cells were subsequently stimulated with either 10 or $100 \mathrm{ng} / \mathrm{ml}$ of IGF-I; control cells did not receive IGF-I stimulation. Cell viability was determined at 24,48 and $72 \mathrm{~h}$ poststimulation using the XTT assay. The results are representative of three experiments and are shown with standard deviation. (C) Phosphorylation of Akt upon IGF-I stimulation ( $100 \mathrm{ng} / \mathrm{ml})$ of serum starved DAOY cells for $2 \mathrm{~min}, 10 \mathrm{~min}, 1 \mathrm{~h}$ and $24 \mathrm{~h}$. Lysates were collected and electrophoresed on a $10 \%$ reducing polyacrylamide gel. Proteins were transferred onto nitrocellulose and the blots were probed with anti-phospho-Akt $\left(\mathrm{Ser}^{473}\right)$ and total Akt. (D) DAOY cells were treated with IGF-I $(100 \mathrm{ng} / \mathrm{ml})$ for $24 \mathrm{~h}$ followed by exposure to LY294002 $(25 \mu \mathrm{M})$ or PD98059 $(50 \mu \mathrm{M})$ for $2 \mathrm{~min}$, $10 \mathrm{~min}, 1 \mathrm{~h}$ and $24 \mathrm{~h}$. Lysates were collected and Western blotting was carried out using anti-phospho-FOXO1 $\left(\operatorname{Ser}^{256}\right)$. the PI3K/Akt pathway $(20,21)$. The human medulloblastoma cell line, DAOY, expresses IGF-IR suggesting the potential for these cells to respond to IGF-I (Fig. 3A). The DAOY cells express almost equivalent levels to that of the rat glioma cell line, C6LacZ, which was used as a positive control for IGF-IR (22); IGF-IR expression was also observed in the UW228-3 human medulloblastoma cell line (data not shown). The IGF-IR in these cells is functional as IGF-I can significantly increase the viability of serum-starved DAOY in response to IGF-I stimulation (Fig. 3B). When the DAOY cell line was cultured in serum-free medium for $48 \mathrm{~h}$ prior to stimulation with IGF-I (10 and $100 \mathrm{ng} / \mathrm{ml}$ ) for 24, 48 and $72 \mathrm{~h}$, IGF-I rescued DAOY cells in a dose-dependent manner. These results indicate that IGF-I can lead to survival of medulloblastoma cells under low-serum conditions.

Treatment with IGF-I was associated with a rapid phosphorylation of Akt within 2 min of stimulation with IGF-I $(100 \mathrm{ng} / \mathrm{ml})$ and which was sustained for at least $24 \mathrm{~h}$ postIGF-I stimulation (Fig. 3C). Of interest, IGF-I induces rapid FOXO1 phosphorylation (Fig. 3D), which is sensitive to treatment with the PI3K/Akt inhibitor LY294002, but insensitive to the Ras-MAPK inhibitor, PD98059, as unphosphorylated FOXO1 appears within 10 min of LY294002 inhibitor administration while it remains phosphorylated in the presence of PD98059 (Fig. 3D). Thus, IGF-I rescues DAOY cells from serum starvation, possibly through the downstream phosphorylation of FOXO1.

Activated FOXO1-AAA significantly suppresses medulloblastoma viability. A mutant form of FOXO1 (FOXO1AAA), containing three modified Akt phosphorylation sites (Ser, Thr to Ala), cannot be negatively regulated by Akt. Thus, adenoviral expression of FOXO1-AAA was utilized to restore FOXO1 activity to DAOY cells in an attempt to ascertain the effects of constitutively active FOXO1 on medulloblastoma viability. DAOY cells were first serum starved for $48 \mathrm{~h}$ prior to treatment with IGF-I for $24 \mathrm{~h}$. These cells were then exposed to either Ad-FOXO1-AAA (Ad-A3) or Ad-LacZ at MOIs (multiplicity of infection) of 100 and assessed for viability after 24, 48 and $72 \mathrm{~h}$ [all DAOY cells are transduced at this MOI (Fig. 4A)]. Cells infected with
A

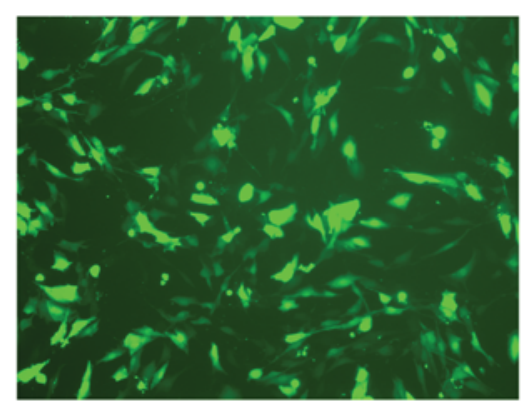

B

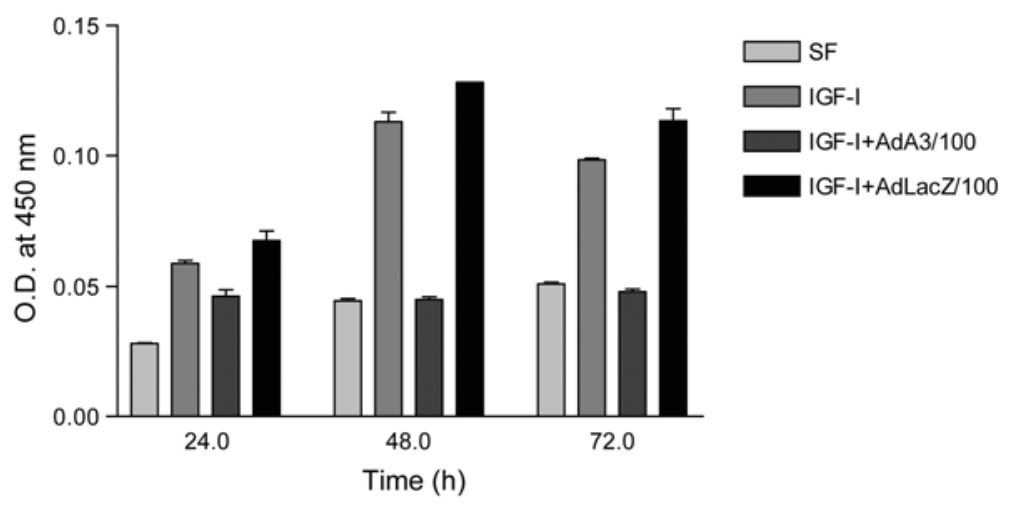

Figure 4. Transduction of medulloblastoma cells with Ad-FOXO1-AAA abrogates the survival effect of IGF-I. (A) Bright-field/GFP overlay of DAOY medulloblastoma cells transduced with Ad-FOXO1-AAA at multiplicity of infection (MOI) 100 for 24 h. (B) Graphical representation of Ad-FOXO1-AAA transduction of IGF-I treated DAOY cells; bars represent mean \pm SEM from at least three experiments. DAOY cells were plated at $2.5 x 10^{4}$ cells per well and buffer exchanged into SF medium for $48 \mathrm{~h}$ prior to IGF-I treatment for $24 \mathrm{~h}$. Adenovirus infection was then carried out with either FOXO1-AAA or LacZ expression cassettes at MOIs of 100 and assessed for viability after 24, 48 and $72 \mathrm{~h}$. 
A

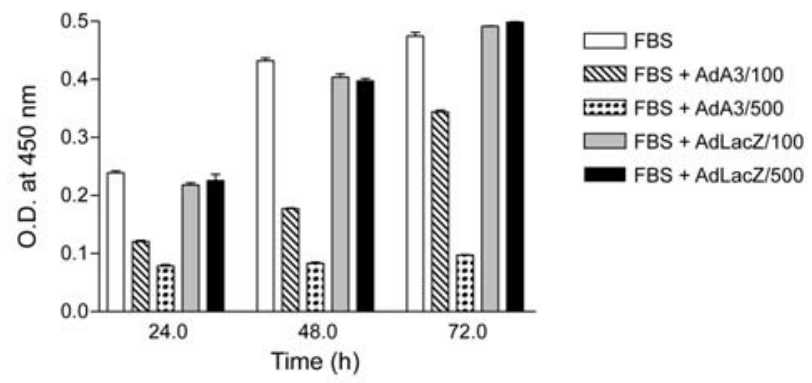

B

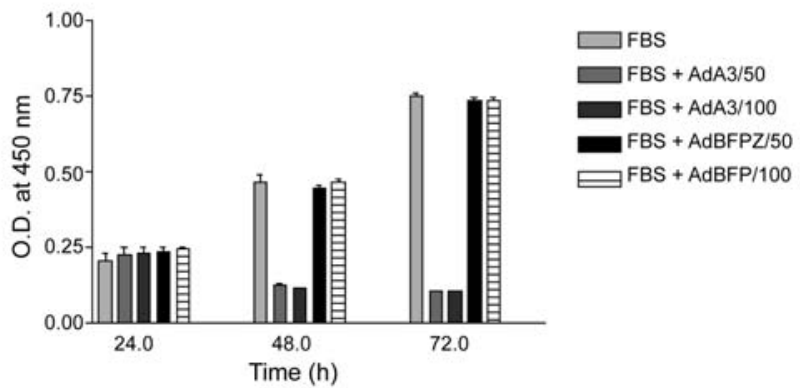

Figure 5. Effect of Ad-FOXO1-AAA transduction on survival of medulloblastoma cells cultured in serum. Graphical representation of Ad-FOXO1AAA treatment of (A) DAOY and (B) UW228-3 medulloblastoma cells grown in complete serum; bars represent mean \pm SEM from at least three experiments. Cells were plated at $2.5 \times 10^{4}$ cells per well and adenoviral infection was then carried out with FOXO1-AAA, LacZ or BFP cassettes at the indicated MOIs and assessed for viability after 24, 48 and $72 \mathrm{~h}$.

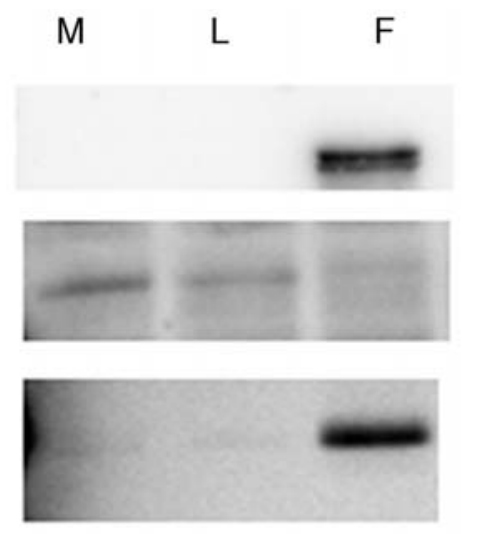

\section{FOXO1}

\section{Cyclin D1}

p27

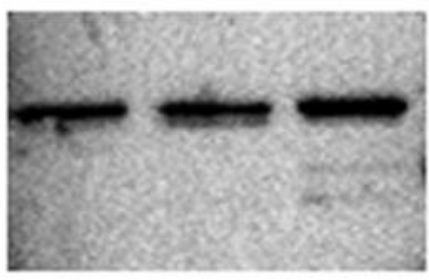

Caspase 9

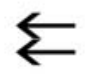

Actin

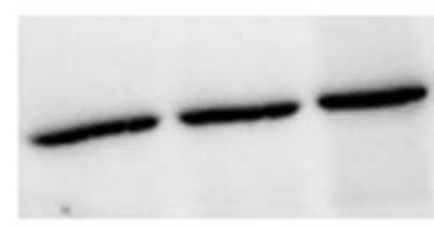

Ad-LacZ showed similar viability to that of mock-infected cells (Fig. 4B). However, the viability of Ad-FOXO1-AAA infected DAOY was significantly reduced compared to that of both Ad-LacZ treated and mock-infected cells (Fig. 4B). Interestingly, Ad-FOXO1-AAA infected DAOY which were exposed to complete serum displayed a similar trend. Cells that were infected with Ad-LacZ at MOIs of 100 and 500 showed similar viability to mock-infected cells (Fig. 5A). In contrast, the viability of Ad-FOXO1-AAA infected DAOY grown in complete serum was significantly reduced at both MOIs as compared to cells that were infected with Ad-LacZ (Fig. 5A). Furthermore, Ad-FOXO1-AAA infections carried out at MOI 500 were able to reduce DAOY viability constantly over a 3-day period. Similarly, viability of UW228-3 medulloblastoma cells growing in complete serum significantly decreased after Ad-FOXO1-AAA transduction at MOIs of 50 and 100 (Fig. 5B). In cells transduced with Ad-FOXO1AAA, Western blot analysis of selected FOXO1 targets showed an increase in the cyclin-dependent kinase inhibitor p27 and a concomitant decrease in cyclin D1 (and cyclin D2, data not shown) as compared to cells transduced with AdLacZ or mock treated (Fig. 6). Importantly, only Ad-FOXO1AAA-transduced cells had activated caspase 9 (Fig. 6). Thus, a FOXO1 construct that cannot be negatively regulated by Akt is a critical direct regulator of medulloblastoma cell survival in the absence or presence of growth factors.

\section{Discussion}

Data from studies in nematode and mammalian cells suggest that regulation of the Forkhead family of transcription factors is key to many cellular responses which include apoptosis, cell cycle function, stress and metabolic responses $(17,23)$. The major targets of the serine-threonine kinase Akt are the Forkhead factors; however there is a dearth of data regarding the involvement of the IGF-I-FOXO1 axis in the survival of medulloblastoma. Here we report that retention of FOXO1 in the cytoplasm is key to medulloblastoma survival and that enforced expression of a constitutively active FOXO1 can dramatically reduce medulloblastoma cell viability. It has been shown that IGF-I can stimulate FOXO3a/FKHRL1 phosphorylation in neuronal cells via the PI3K-Akt pathway thus inhibiting this pro-apoptotic transcription factor (24). The common mechanism for inhibition has been attributed to phosphorylation of the FOXO factors via insulin or other growth factor signaling cascades leading to their retention in the cytoplasm and thus repression of pro-apoptotic genetic programs (17). For example, in cerebellar granule cells, the ultimate phenotypic response for FOXO regulation by the IGF-I signaling cascade is the blockade of FOXO3a/FKHRL1dependent transcription of Bim, which is a principal player in initiating the intrinsic (mitochondrial) apoptotic pathway (25). Furthermore, nuclear exclusion of FOXO1 induced via phosphorylation by insulin serves to target FOXO1 for ubiquitin-mediated degradation (26). We have shown for the first time in medulloblastoma cells that FOXO1 is phosphorylated upon sole IGF-I stimulation subsequent to serum starvation (Fig. 3D). Furthermore, IGF-I-induced FOXO1 phosphorylation appears to be PI3K-dependent rather

Figure 6. Effect of Ad-FOXO1-AAA transduction on selected FOXO1 targets. UW228-3 cells were infected at MOI 100 for $24 \mathrm{~h}$ prior to cell lysis and Western blot analysis of FOXO1, cyclin D1, p27 and caspase 9; actin was used as a loading control. Abbreviations: M, mock (PBS); L, Ad-LacZ; F, Ad-FOXO1-AAA.

\begin{abstract}
than dependent on the MAPK pathway. Thus, in the medullo-
\end{abstract}


blastoma cells, integrity of the PI3K-Akt pathway is required for FOXO1 phosphorylation, in accordance with previous findings in other cell types $(17,23,24,26,27)$.

Upon serum starvation of the medulloblastoma cells, the subcellular localization of FOXO1 was primarily nuclear. This observation is in agreement with the commonly held hypothesis that in the absence of IGF-I signaling FOXO1 is no longer phosphorylated and thus can be imported into the nucleus to effect target gene expression. Serum starvation led to a drastic reduction in medulloblastoma cell viability (Fig. 1). The findings of nuclear FOXO1 localization and low medulloblastoma viability upon serum starvation prompted us to investigate the link between the regulation of FOXO1 status and medulloblastoma survival. Since phosphorylated FOXO1 has been hypothesized to lead to cell survival, a dominant-negative form of FOXO1 (FOXO1-AAA), in which the three Akt phosphorylation sites are altered, was used to test the null hypothesis that medulloblastoma cell death requires FOXO1 to be constitutively non-phosphorylated in the presence of growth factor signaling. We report here that adenovirus-mediated delivery of FOXO1-AAA into medulloblastoma cells can reduce cell viability (Figs. 4-6), which is in keeping with other published reports demonstrating a regulatory role for FOXO1 in apoptosis (28-31). Indeed, a recent study demonstrated that pharmacological inhibition of the p85 subunit of PI3K in human breast carcinoma cells leads to activation of FOXO transcription factors and subsequent transcriptional induction of cell cycle arrest and apoptotic genes (32). As well, dramatic apoptosis of LAPC4 prostate carcinoma cells occurred upon adenovirus-mediated overexpression of FOXO1 and FOXO3a (33). Interestingly, in our study, Ad-FOXO1-AAA treatment significantly abrogated medulloblastoma viability in the presence of complete serum (Figs. 5 and 6), which suggests that FOXO1 may represent an important convergence point for multiple signaling pathways.

Taken together, the data presented here support a novel role for FOXO1 in medulloblastoma viability and furthermore, demonstrate that adenoviral expression of a mutant form of FOXO1 (FOXO1-AAA), which cannot be phosphorylated, can induce efficient growth suppression of medulloblastoma cell lines. Thus, means of inhibiting FOXO1 nuclear export (34) might provide important therapeutic applications, allowing the activity of a single downstream transcription factor, such as FOXO1, to overcome defects in tyrosine kinase receptor signalling through the PI3K-Akt pathway.

\section{Acknowledgements}

We thank W. Sellers and R. Del Maestro for reagents and cells. V.K.S. was supported by scholarships from the Montreal Center for Experimental Therapeutics in Cancer (MCETC) and the Fonds de la recherche en santé du Québec (FRSQ). J.N. was a National Scholar of the FRSQ. This work was funded by the National Cancer Institute of Canada through the Canadian Cancer Society.

\section{References}

1. Paulino AC: Current multimodality management of medulloblastoma. Curr Probl Cancer 26: 317-356, 2002.
2. Whelan HT, Krouwer HG, Schmidt MH, Reichert KW and Kovnar EH: Current therapy and new perspectives in the treatment of medulloblastoma. Pediar Neurol 18: 103-115, 1998.

3. Modha A, Vassilyadi M, George A, Kuehn S, Hsu E and Ventureyra ECG: Medulloblastoma in children - the Ottawa experience. Child's Nerv Syst 16: 341-350, 2000.

4. Polkinghorn WR and Tarbell NJ: Medulloblastoma: tumorigenesis, current clinical paradigm, and efforts to improve risk stratification. Nat Clin Pract Oncol 4: 295-304, 2006.

5. Kiltie AE, Lashford LS and Gattamaneni HR: Survival and late effects in medulloblastoma patients treated with craniospinal irradiation under three years old. Med Pediatr Oncol 28: 348-354, 1997.

6. Corcoran RB and Scott MP: A mouse model for medulloblastoma and basal cell nevus syndrome. J Neurooncol 53: 307-318, 2001

7. Pomeroy SL, Tamayo P, Gaasenbeek M, et al: Prediction of central nervous system embryonal tumour outcome based on gene expression. Nature 415: 436-442, 2002.

8. Cogen PH, Daneshvar L, Metzger AK, Duyk G, Edwards MS and Sheffield VC: Involvement of multiple chromosome 17p loci in medulloblastoma tumorigenesis. Am J Hum Genet 50: 584-589, 1992.

9. Goodrich LV, IMG SRC, Higgins KM and Scott MP: Altered neural cell fates and medulloblastoma in mouse patched mutants. Science 277: 1109-1113, 1997.

10. Rao G, Pedone CA, Valle LD, Reiss K, Holland EC and Fults DW: Sonic hedgehog and insulin-like growth factor signaling synergize to induce medulloblastoma formation from nestin-expressing neural progenitors in mice. Oncogene 23: 6156-6162, 2004.

11. Wang JY, Del Valle L, Gordon J, et al: Activation of the IGF-IR system contributes to malignant growth of human and mouse medulloblastomas. Oncogene 20: 3857-3868, 2001.

12. Hartmann W, Gon-Sontgerath B, Koch A, et al: Phosphatidylinositol 3 '-kinase/AKT signaling is activated in medulloblastoma cell proliferation and is associated with reduced expression of PTEN. Clin Cancer Res 12: 3019-3027, 2006.

13. Wlodarski P, Grajkowska W, Rainko K and Jozwiak J: Activation of Akt and Erk pathways in medulloblastoma. Folia Neuropathol 44: 214-220, 2006.

14. Hambardzumyan D, Becher OJ, Rosenblum MK, Pandolfi PP, Manova-Todorova K and Holland EC: PI3K pathway regulates survival of cancer stem cells residing in the perivascular niche following radiation in medulloblastoma in vivo. Genes Dev 22: 436-448, 2008.

15. Arden KC: Multiple roles of FOXO transcription factors in mammalian cells point to multiple roles in cancer. Exp Gerontol 41: 709-717, 2006.

16. Calnan DR and Brunet A: The FoxO code. Oncogene 27: 2276-2288, 2008.

17. Burgering BMT and Kops GJPL: Cell cycle and death control: long live Forkheads. TIBS 27: 352-360, 2002.

18. Keles GE, Berger MS, Srinivasan J, Kolstoe DD, Bobola MS and Silber JR: Establishment and characterization of four human medulloblastoma-derived cell lines. Oncol Res 7: 493-503, 1995.

19. He TC, Zhou S, da Costa LT, Yu J, Kinzler KW and Vogelstein B: A simplified system for generating recombinant adenoviruses. Proc Natll Acad Sci USA 95: 2509-2514, 1998.

20. Sachdev D and Yee D: Disrupting insulin-like growth factor signaling as a potential cancer therapy. Mol Cancer Ther 6: $1-12,2007$.

21. Valentinis B and Baserga R: IGF-I receptor signalling in transformation and differentiation. Mol Pathol 54: 133-137, 2001.

22. Resnicoff M, Burgaud JL, Rotman HL, Abraham D and Baserga R: Correlation between apoptosis, tumorigenesis, and levels of insulin-like growth factor I receptors. Cancer Res 55: 3739-3741, 1995.

23. Kops GJPL, Dansen TB, Polderman PE, et al: Forkhead transcription factor $\mathrm{FOXO} 3$ a protects quiescent cells from oxidative stress. Nature 419: 316-321, 2002.

24. Zheng WH, Kar S and Quirion R: Insulin-like growth factor-1induced phosphorylation of the forkhead family transcription factor FKHRL1 is mediated by Akt kinase in PC12 cells. JBC 275: 39152-39158, 2000. 
25. Linseman DA, Phelps RA, Bouchard RJ, et al: Insulin-like growth factor-I blocks Bcl-2 interacting mediator of cell death (Bim) induction and intrinsic death signaling in cerebellar granule neurons. J Neurosci 22: 9287-9297, 2002.

26. Matsuzaki H, Daitoku H, Hatta M, Tanaka K and Fukamizu A: Insulin-induced phosphorylation of FKHR (Foxo1) targets to proteasomal degradation. Proc Natl Acad Sci USA 100: $11285-11290,2003$

27. Nakamura N, Ramaswamy S, Vazquez F, Signoretti S, Loda M and Sellers WR: Forkhead transcription factors are critical effectors of cell death and cell cycle arrest downstream of PTEN Mol Cell Biol 20: 8969-8982, 2000.

28. Brunet A, Bonni A, Zigmond MJ, et al: Akt promotes cell survival by phosphorylating and inhibiting a forkhead transcription factor. Cell 96: 857-868, 1999.

29. Nakamura T and Sakamoto K: Forkhead transcription factor FOXO subfamily is essential for reactive oxygen speciesinduced apoptosis. Mol Cell Endocrinol 281: 47-55, 2008.

30. Ramaswamy S, Nakamura N, Sansal I, Bergeron L and Sellers WR: A novel mechanism of gene regulation and tumor suppression by the transcription factor FKHR. Cancer Cell 2: $81-91,2002$
31. Tang ED, Nunez G, Barr FG and Guan KL: Negative regulation of the forkhead transcription factor FKHR by Akt. J Biol Chem 274: 16741-16746, 1999.

32. Reagan-Shaw S and Ahmad N: RNA interference-mediated depletion of phosphoinositide 3-kinase activates forkhead box class $\mathrm{O}$ transcription factors and induces cell cycle arrest and apoptosis in breast carcinoma cells. Cancer Res 66: 1062-1069, 2006.

33. Modur V, Nagarajan R, Evers BM and Milbrandt J: FOXO proteins regulate tumor necrosis factor-related apoptosis inducing ligand expression. Implications for PTEN mutation in prostate cancer. J Biol Chem 277: 47928-47937, 2002.

34. Kau TR, Schroeder F, Ramaswamy S, et al: A chemical genetic screen identifies inhibitors of regulated nuclear export of a Forkhead transcription factor in PTEN-deficient tumor cells. Cancer Cell 4: 463-476, 2003. 